\title{
Diseño de un esquema de relaciones para UN SISTEMA DE INFORMACIÓN NORMATIVO EN UNA INSTITUCIÓN DE EDUCACIÓN SUPERIOR
}

\author{
Álvaro Monterroza Ríos ${ }^{1}$ \\ DARÍo HURTADO ${ }^{2}$
}

\section{Resumen}

El artículo presenta el desarrollo de un esquema de relaciones que sirve de soporte para un sistema de información normativo. Se parte desde el ámbito de la gestión del conocimiento y gestión de la información para formular las relaciones de las diferentes áreas de una organización de educación superior con criterios del ordenamiento jurídico colombiano y la gestión por procesos. Para el primer criterio se toma el esquema de jerarquía jurídica que posee el estado colombiano y para el segundo los conceptos de la "Gestión por Procesos" recomendada por la ISO 9000:2000. La propuesta se concreta en un esquema relacional normativo, es decir, una forma de relacionar el marco jurídico con cada instancia (procesos y áreas) para cualquier Institución de Educación Superior organizada por procesos.

1 Ingeniero Químico, Magíster en Ingeniería Química, estudiante de Filosofía. Actualmente se desempeña como docente e investigador del Instituto Tecnológico Metropolitano de Medellín. Grupo de Investigación en Gestión Administrativa (GIGA).

2 Administrador de Empresas, Especialista en Gerencia. Actualmente se desempeña como docente e investigador del Instituto Tecnológico Metropolitano de Medellín. Grupo de Investigación en Gestión Administrativa (GIGA).

Fecha de recepción: 30 de abril de 2008

Fecha de aceptación: 11 de agosto de 2008 


\section{Palabras clave}

Conocimiento organizacional, Sistema de Información, Normatividad, Gestión del conocimiento, Gestión por procesos.

\section{Alostract}

This paper presents a relation schema that gives support to a normative information system. The starting point is the knowledge and information management domain to formulate the relations of different areas of a higher education organization with criteria taken from the Colombian legislation and process management. The first criterion is taken from the Colombian legal hierarchy schema and, the second, is taken from the concepts of "process management" recommended by the ISO 9000:2000 standard. The proposal is set in a normative relational schema, that is, a way to relate the legal frame with each instance (processes and areas) for any higher education institution organized by processes.

\section{Key words}

Organizational knowledge, Information System, Laws, Information Management, Legal Order. 


\section{INTRODUCCIÓN}

El rol del conocimiento en las organizaciones ha cambiado principalmente porque se ha hecho evidente el concepto de valor de éstas están en el saber hacer las cosas, es decir, su conocimiento organizacional. Como se muestra a continuación, el buen manejo de la información es una actividad esencial para mejorar el aprendizaje organizacional y crear capital intelectual.

Los sistemas de información, dentro del ámbito de la "gestión del conocimiento", se han convertido en herramientas importantes para la gestión, la administración y la gerencia dentro de una organización, ya que, precisamente, la gestión de la información significa contar con información pertinente, precisa y en el momento oportuno, que permita generar coordinación, conectividad y trabajo en equipo.

Se puede partir de las preguntas: ¿Cómo es posible diseñar un sistema que mejore la gestión de la información en las instituciones de educación superior, en términos de pertinencia, accesibilidad, seguridad y confiabilidad? Y, de ser posible, ¿de qué manera logrará este desarrollo mejorar la efectividad organizacional de las instituciones? El propósito de este artículo es, justamente, diseñar un esquema relacional ${ }^{3}$, es decir, un modelo esquemático que permita abarcar a toda la organización desde sus procesos e interrelacionarlos entre sí con componentes taxonómicos ${ }^{4}$ para la construcción de un sistema de información normativo válido para cualquier Institución de Educación Superior (IES), que trabaje por procesos relacionados con la gestión administrativa de una organización.

3 Aunque en varios contextos se utiliza el adjetivo "relacional" para los modelos basados en la lógica de predicado y en la teoría de conjuntos, en nuestro caso sólo lo denominamos "relacional" en el sentido de las relaciones creadas entre las normas jurídicas y los distintos procesos y áreas de una organización.

4 Taxonomía en este contexto la denotaremos como un sistema de clasificación compuesto por una jerarquía. Viene del griego $\tau a \xi \iota \varsigma$, taxis, "ordenamiento", y vouos, nomos, "norma" o "regla". 
Con esta propuesta de sistema de información normativo se podrán dar las bases para una herramienta efectiva para el control interno, la comunicación permanente entre las distintas áreas de las IES y para la adecuada y oportuna toma de decisiones acerca de cada uno de sus procesos.

\section{MARCO TEÓRICO}

\subsection{Conocimiento organizacional}

Los sistemas de información son herramientas administrativas para plasmar las huellas que deja el conocimiento organizacional o también las condiciones que éste necesita. Un sistema de información para una organización debe situarse en el contexto de lo que se conoce "gestión del conocimiento", ya que los fines de la gestión de la información son, en última instancia, el buen manejo del conocimiento y hacer de la información el insumo básico y fundamental para la competitividad organizacional.

En la práctica y en los desarrollos teóricos del ámbito de la gestión del conocimiento es difícil unificar el concepto de "conocimiento", ya que se entiende de muchas formas. Álava y Leader (1999) dan a conocer las diversas formas en que se puede entender, y por estas divergencias, hace que su estudio sea complejo y variado en el ámbito organizacional. Según estos autores, el conocimiento se puede entender de múltiples formas: como datos e información, como estados mentales, como también un objeto factible de almacenar y manipular. Por otro lado, también se le entiende como un proceso transformador de experiencias, una condición de acceso a la información o simplemente como una capacidad o competencia. Cada uno de estos acercamientos, si bien son parcialmente ciertos, no describen plenamente lo que es el conocimiento, y mucho menos lo que es el conocimiento organizacional.

En nuestra propuesta se va a estudiar el conocimiento sólo en el ámbito organizacional, ya que partimos del presupuesto que las instituciones también pueden aprender, pues las entidades exitosas son las que hacen del conocimiento su capital organizacional y un 
factor diferenciador y gran generador de valor, es decir, son "organizaciones inteligentes". Aunque señalemos el conocimiento en varios contextos, nuestro acercamiento será en la forma en que aprenden las organizaciones. En otras palabras, existen varios enfoques a este estudio (como el ámbito filosófico, epistemológico, científico, de la ingeniería o de la contaduría), pero los tomaremos de forma complementaria en el estudio dentro del campo del conocimiento organizacional.

Con base en esta aclaración, adoptaremos la concepción de conocimiento que desarrolló el profesor Guillermo Correa de la Escuela Interamericana de Bibliotecología de la Universidad de Antioquia, que para nuestro parecer, recoge los diferentes acercamientos sobre el conocimiento y los sintetiza en la siguiente definición (Correa, 2007):

(el conocimiento es)... el resultado de un proceso de interacción mental (percepción -reflexión-interpretación) sinérgico, en un momento dado de información, habilidades, creencias, modelos mentales, destrezas y experiencias en un contexto situado, realizado por una persona, al tomar una decisión, que conlleva una acción, con el fin de resolver un problema o satisfacer una necesidad y contribuir así al desarrollo humano.

Esta definición dice muchas cosas que desglosaremos con detalle. Lo primero es que el conocimiento es una interacción mental, por tanto requiere de personas o sujetos para llegar a crearse, lo que está de acuerdo con los postulados de Polanyi (1966) acerca de que el conocimiento es personal, pero puede actuar sinérgicamente con otras mentes.

Otro elemento importante es que el conocimiento no puede ser sólo información, sino que es sólo una de las condiciones para que éste se dé, es decir, también se requiere de las habilidades, creencias y modelos mentales de un sujeto para que éste se lleve a cabo. Esto quiere decir que el conocimiento no sólo necesita de información o datos para que exista, sino que se considera también la "carga teórica" (habilidades, creencias y modelos mentales) que cada sujeto lleva consigo y que está retroalimentándose permanentemente cada 
vez que surge un conocimiento nuevo. Esta postura es similar a las tesis epistemológicas de Hanson (edición en español, 1977), que afirma que uno sólo ve con el concepto (teorías y modelos mentales) y que la experiencia (información y datos del exterior) son la "ocasión" para que se surja el conocimiento.

Siguiendo con la definición de Correa sobre el conocimiento organizacional, también notamos que éste debe ser puesto en un contexto determinado, es decir, el conocimiento es la solución, un "destello" para una determinada situación y que inmediatamente sale y desaparece.

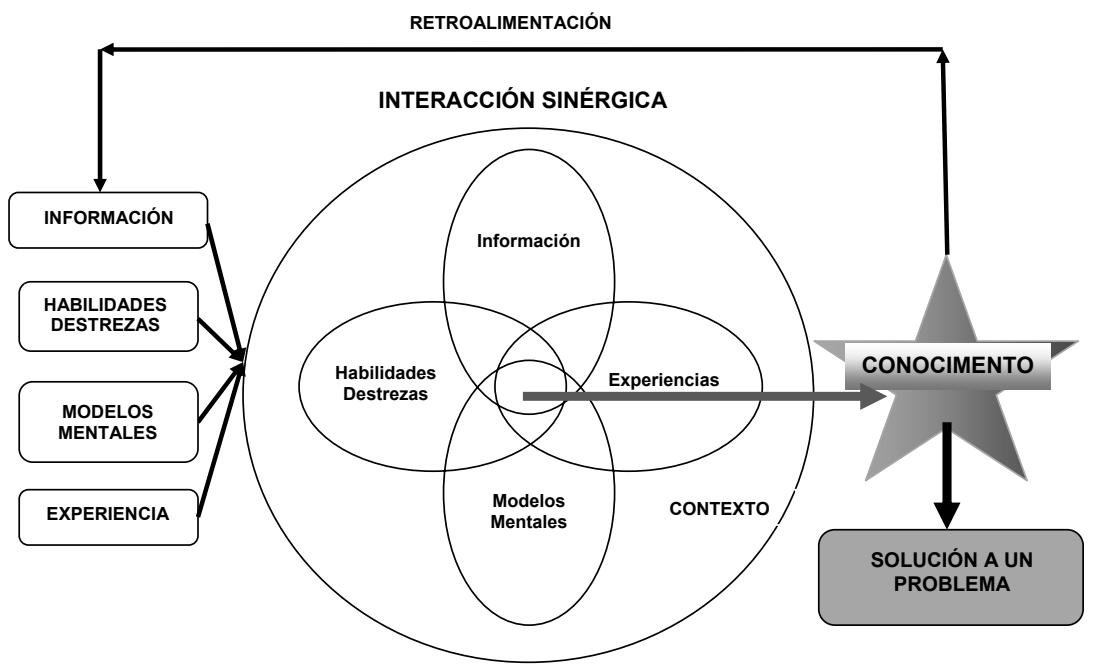

Figura 1. Modelo de ADquisición de Conocimiento (Correa, 2007)

Del conocimiento sólo quedan las huellas que pueden ser recogidas parcialmente en información, pero también surgen nuevas habilidades, destrezas, experiencias o creencias y pueden cambiar los modelos mentales. Como se muestra en la figura 1, el modelo es la chispa que da la solución a un determinado problema.

Esto nos permite concluir, y tal como se visualiza en el esquema, es un proceso sinérgico ya que el resultado es mayor que los resultados obtenidos de la suma de los componentes (Correa, 2007). 


\subsection{Conocimientos explícito y tácito en el ámbito organizacional}

Los conceptos de conocimientos tácito y explícito pueden ser muy útiles en el ámbito organizacional debido a que dan pautas para distinguir y conectar el conocimiento personal, del conocimiento que se genera en las interacciones de una organización. Estos términos lo usaron Nonaka y Takeuchi (1995), explicando que el conocimiento organizacional es un proceso de interacción entre conocimiento tácito y explícito que tiene naturaleza dinámica y continua. Se constituye en una espiral permanente de transformación interna del conocimiento (ver figura 2).

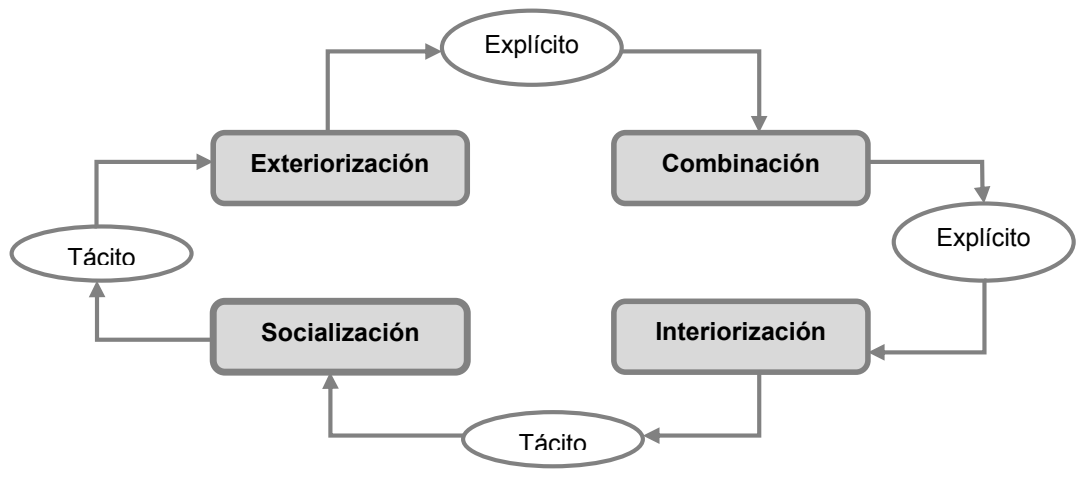

Figura 2. Modelo Nonaka y TAKeuchi (1995)

Según Soto (2006), este modelo es el más conocido y aceptado de creación de conocimiento y se expresa un mecanismo en el cual el conocimiento se genera mediante dos espirales de contenido: epistemológico y ontológico. La espiral ontológica se refiere a las cuatro fases allí manifestadas (figura 2); mientras que el espiral epistemológico, lo conforman las uniones entre el conocimiento tácito y el conocimiento explícito.

Este modelo plantea que las empresas innovan mediante la transformación del conocimiento individual (tácito), en conocimiento organizacional (explícito). "Se fundamenta en las interrelaciones 
producidas entre las dimensiones epistemológica y ontológica, que originan un modelo de espiral de conocimiento creado, mediante la interrelación de las diferentes formas de conversión del conocimiento: socialización, exteriorización, interiorización y combinación" (Soto, 2006).

Sin embargo, estos conceptos son originalmente tomados del filósofo y químico húngaro Michael Polanyi (1966), quien sostiene que existe una división entre un conocimiento articulado asociado al lenguaje (que él llama conocimiento explícito) y un conocimiento tácito o silencioso. Aunque Nonaka y Takeuchi propusieron usar estos conceptos en el ámbito del aprendizaje organizacional.

Retomando la propuesta de Polanyi, el conocimiento explícito tiene su fundamento en un lenguaje articulado el cual puede lograr grandes alcances dependiendo de la disposición y acceso a la información que tenga cada individuo. El conocimiento tácito, por el contrario, se alcanza sin necesidad de un lenguaje y con el cual logramos interactuar con el entorno. Este conocimiento es similar al que tienen los animales sobre su entorno y con él somos capaces de realizar nuestras necesidades básicas para la supervivencia.

El conocimiento explícito se aprende por un conjunto de operaciones sistemáticas y puede ser extendido enormemente siguiendo determinados patrones de aprendizaje. En cambio, el conocimiento tácito sólo se aprende por error y ensayo, según Polanyi, es una "reorganización de la experiencia".

Polanyi asegura que estas afirmaciones tienen interesantes consecuencias: una de ellas es que el conocimiento tácito es mucho más limitado que el conocimiento articulado; otra es que estos tipos de conocimiento tienen una diferencia lógica esencial; el conocimiento explícito puede ser crítico ya que se puede reflexionar sobre sí mismo, mientras que el tácito está condenado a ser aceptado tal como lo aprendimos, o sea en forma acrítica.

¿Cómo relacionar entonces el conocimiento tácito y explícito en el ámbito organizacional? Consideramos tomar la vía del profesor Guillermo Correa que asocia el conocimiento organizacional tácito con el conocimiento personal de cada individuo dentro de una 
institución y el conocimiento explícito, como el conocimiento propio que genera una organización. Cabe mencionar que nace el concepto de cultura institucional ${ }^{5}$.

Por tanto, Correa hace una adecuación del modelo de adquisición de conocimiento mostrado en la figura 1 , que se hace referencia a continuación (figura 3).

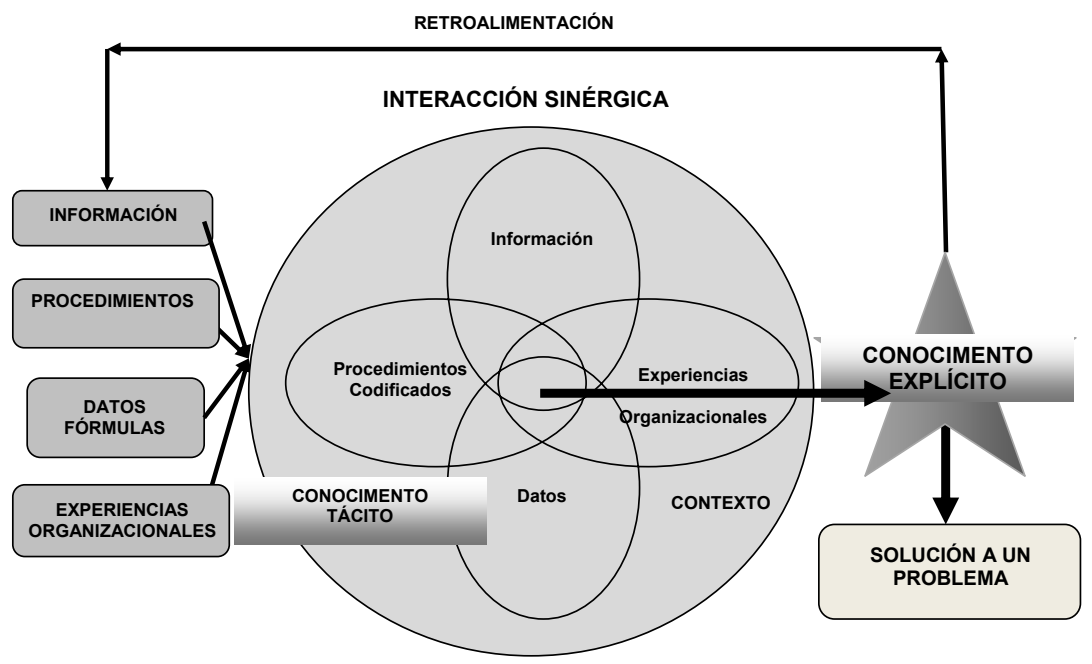

Figura 3. Modelo de ADQUISICIÓN DE CONOCIMIENTO EXPLÍCITO ORGANIZACIONAL

(CORREA, 2007)

El cambio radica en que esta vez no sucede dentro de los sujetos individuales, sino dentro de las organizaciones. La "carga teórica" de una institución será distinta: las habilidades y destrezas de un sujeto se cambiarían por procedimientos codificados; los modelos

5 La cultura "es el patrón general de conducta, creencias y valores que sus miembros comparten. Se le puede inferir de lo que la gente dice, hace y piensa en el contexto de una organización. Implica la adquisición y transmisión de conocimientos, creencias y patrones de conducta en el transcurso del tiempo, lo que significa que la cultura de una organización es sumamente estable y no cambia rápidamente" (Koontz y Weihrich, xxx, p. 326). 
mentales, por datos y fórmulas; y las experiencias personales, por experiencias organizacionales. Los otros elementos y conexiones del modelo de conocimiento personal, se mantienen prácticamente iguales.

Si el conocimiento explícito se puede aprender por un conjunto de operaciones sistemáticas y puede ser extendido siguiendo determinados patrones de aprendizaje, una organización puede aprender. Por tanto, afirma Correa, la información, los procedimientos codificados, la experiencia y los datos son las condiciones para que exista o nazca el conocimiento explícito en la organización.

Lo que se busca entonces con un sistema de información no es un almacenamiento de conocimiento, ya que éste no se puede guardar sin convertirse en información, sino que busca crear las condiciones (información, procedimientos, experiencias y datos organizacionales) para que el conocimiento surja y dé solución a un determinado problema. Consecuentemente, retroalimentará a la misma aumentando su aprendizaje y capital intelectual. Dentro del conocimiento explícito de una organización se encuentran las directrices, políticas y estrategias de direccionamiento que conforman su marco normativo,

El sistema de información de normas es una de varias herramientas de información necesarias para crear las condiciones que mejoran el aprendizaje organizacional, especialmente en una institución de educación superior en que sus marcos regulativos jurídicos le dan forma, carácter y obligatoriedad en su naturaleza misma.

\subsection{Gestión de la información y gestión del conocimiento}

Como hemos mencionado en la sección anterior, la propuesta de un sistema de información está enmarcada dentro de la gestión del conocimiento en la medida en que si se gestiona la información, se crea una de las más importantes condiciones para lograr conocimiento organizacional. Sin embargo, es bueno aclarar que existen diferencias conceptuales importantes si estos sistemas sólo llegan a gestionar la información y su objetivo no va orientado 
hacia la gestión del conocimiento. Por esto, retomamos un paralelo entre lo que es gestión de la información y lo que se quiere con la gestión del conocimiento.

En casi todas las referencias sucede que la gestión de la información y la gestión del conocimiento están estrechamente vinculadas. Según Ponjuán, "No resulta fácil y tal vez no sea totalmente visible la frontera entre algunas facetas de la Gestión de la Información (GI) y la Gestión del Conocimiento (GC)". La autora aclara que acerca de este tema se manejan múltiples enfoques, y por ser un campo aún en exploración, muchas de sus bases teóricas y metodológicas están por definirse (Ponjuán, 2004)

De forma similar, Soto y Barrios (2005) presentan una cita en la que definen la gestión de la información, como "el manejo del conocimiento adquirido por una o varias fuentes diferentes, para mejorar el acceso a todos los que tengan que compartir o utilizar dicho conocimiento". Según las autoras, "esta definición expresa claramente la relación que existe entre la gestión de la información y la gestión del conocimiento y corrobora, una vez más, que sus fronteras no están definidas y que ambas actividades se encuentran vinculadas", por esto afirman que la una forma parte de la otra. De otro lado, Bustelo y Amarillas hacen una definición más precisa y afirman que la gestión de la información es "el conjunto de las actividades que se realizan con el propósito de adquirir, procesar, almacenar y finalmente, recuperar de manera adecuada la información que se produce o se recibe en una organización y que permite el desarrollo de sus actividades" (Bustelo y Amarillas, 2001).

Autores como Bustelo y Arguello, citados por Soto y Barrios, aseguran que los desarrollos en la gestión del conocimiento son proyectos de gestión de la información. Sin embargo, Soto y Barrios también citan a Pérez Capdevila y marcan las diferencias que existen, que se presentan a continuación (tabla 1): 
Tabla 1. Diferencias entre los proyectos de Gestión del CONOCIMIENTO Y Gestión DE LA INFORMACIÓN (SOTO Y BARRIOS, 2005)

\begin{tabular}{|l|l|}
\hline \multicolumn{1}{|c|}{$\begin{array}{c}\text { Proyecto de Gestión } \\
\text { del Conocimiento }\end{array}$} & \multicolumn{1}{c|}{$\begin{array}{c}\text { Proyecto de Gestión } \\
\text { de la Información }\end{array}$} \\
\hline $\begin{array}{l}\text { Las metas acentúan el valor } \\
\text { agregado para los usuarios. }\end{array}$ & $\begin{array}{l}\text { Las metas acentúan la liberación y } \\
\text { acceso a la información }\end{array}$ \\
\hline $\begin{array}{l}\text { Apoyen las mejoras operaciones } \\
\text { y la innovación. }\end{array}$ & Apoya las operaciones existentes \\
\hline $\begin{array}{l}\text { Agrega valor al contenido por } \\
\text { medio de filtros, la síntesis, } \\
\text { la interpretación y el recorte } \\
\text { de contenido. }\end{array}$ & $\begin{array}{l}\text { Libera contenidos disponibles con peque- } \\
\text { no valor agregado }\end{array}$ \\
\hline $\begin{array}{l}\text { Frecuentemente requiere contri- } \\
\text { buciones y retroalimentación. }\end{array}$ & $\begin{array}{l}\text { Enfatiza la transferencia de información } \\
\text { en un sentido }\end{array}$ \\
\hline $\begin{array}{l}\text { Enfoque balanceado entre los as- } \\
\text { pectos tecnológicos y culturales. }\end{array}$ & Fuerte enfoque tecnológico \\
\hline $\begin{array}{l}\text { Variaciones en los sistemas de } \\
\text { entrada, no permiten automati- } \\
\text { zar el proceso de captura. }\end{array}$ & $\begin{array}{l}\text { Asume que la captura de información } \\
\text { puede ser automatizada }\end{array}$ \\
\hline
\end{tabular}

A pesar de las diferencias que marcan las autoras, hay dificultades conceptuales en afirmar que existe una real gestión del conocimiento en vez de una gestión de la información. Por esto, el objetivo de la investigación ${ }^{6}$ que generó este artículo es plantear un sistema de información para crear condiciones apropiadas de una cultura alrededor del conocimiento organizacional.

\subsection{Marco jurídico}

No se puede concebir un sistema de información de normas sin considerar el concepto de ordenamiento jurídico. El ordenamiento jurídico es "la suma o conjunto de normas de distinto rango jerárquico que tienen validez y vigencia en un determinado contexto

6 Investigación del grupo GIGA: Grupo de Investigación en Gestión y Administración del Instituto Tecnológico Metropolitano de Medellín. 
histórico, y que se estructuran en grados o niveles subordinadas unas a otras" (Martínez Roldán, 2005, p. 81). En el caso de los estados liberales, el ordenamiento jurídico está formado por su Constitución Nacional, que se edifica como la norma suprema.

En Colombia, la Constitución Política de 1991 determina el ordenamiento jurídico que da origen a todas las leyes y determina la jerarquía que regula los entes públicos y privados. Las normas externas surgen con base en las normas constitucionales de la Carta Magna de un Estado, de allí el legislativo emite leyes y decretos-ley, en una jerarquía decreciente; y teniendo en cuenta los marcos que le imponen las constitución y las leyes del legislativo, el ejecutivo emite decretos presidenciales y normas (ejecutivas). A nivel regional, el legislativo y el ejecutivo de cada departamento emiten leyes hasta llegar a un ámbito local en el que el concejo y la alcaldía de cada municipio dictan las normas de carácter más inmediato a una determinada institución educativa, principalmente si es de carácter pública.

En el ámbito educativo, el ordenamiento jurídico determina el marco normativo interno de cualquier institución. A su vez, estas instituciones dan origen a actos administrativos internos que gozan de la presunción de legalidad y que son expedidos con fundamento en las normas externas de carácter legal (Constitución, leyes entre otras). Entonces, tenemos que para institución educativa existe una normatividad externa que delimita y da forma a su funcionamiento, y una normatividad interna que organiza la institución para cumplir su misión dentro de los marcos legales que le impone la normatividad externa. Se observa la necesidad de administrar eficazmente la información normativa, tanto para el cumplimiento misional de las instituciones como para el apoyo a su gestión y su control.

Una propuesta es el diseño de un buen esquema de relaciones entre distintas áreas o procesos de una organización para la gestión de la información normativa que esté acorde con las necesidades y estructura de las instituciones educativas mencionadas que se ajuste a las nuevas formas de administrar una organización, es decir, una 
forma de organizar un sistema de información que permita localizar fácilmente la información de normas y la interrelación entre ella en un determinado proceso en la organización educativa.

Un modelo para la gestión de la información normativa se considera una herramienta de control adicionalmente a los estipulados por la ley y a los adoptados por las entidades, los cuales están soportados en un modelo taxonómico cuyos pilares se fundamentan en dos grandes columnas: el ordenamiento jurídico constitucional de Colombia y el modelo de gestión por procesos. Según el ordenamiento jurídico de Colombia, un sistema en que se gestione la calidad debe permitir (Congreso de la República de Colombia, Ley 872, 2003):

a) Detectar y corregir oportunamente y en su totalidad las desviaciones de los procesos que puedan afectar negativamente el cumplimiento de sus requisitos y el nivel de satisfacción de los usuarios, destinatarios o beneficiarios de conformidad con las normas que lo regulan.

b) Controlar los procesos para disminuir la duplicidad de funciones, las peticiones por incumplimiento, las quejas, reclamos, denuncias y demandas;

c) Registrar de forma ordenada y precisa las estadísticas de las desviaciones detectadas y de las acciones correctivas adoptadas;

d) Facilitar control político y ciudadano a la calidad de la gestión de las entidades, garantizando el fácil acceso a la información relativa a los resultados del sistema;

e) Ajustar los procedimientos, metodologías y requisitos a los exigidos por normas técnicas internacionales sobre gestión de la calidad.

Un sistema de información normativo es entonces útil para apoyar y llevar a cabo estas sugerencias sobre los requerimientos de un sistema de gestión de la calidad, ya que apoya y facilita el control y la gestión organizacional. 


\subsection{Modelo de gestión por procesos}

Una de las preocupaciones de varios pensadores dentro del movimiento de calidad, es la de mejorar la efectividad de las organizaciones para entregar bienes y servicios que agregaran valor y éste fuera percibido por los clientes. En concordancia con el objetivo que la misión y visión de la institución hubiera fijado, se encuentra que una de las limitaciones más grandes en dicho propósito era la visión fragmentada de operaciones individuales realizadas por unidad organizacionales específica.

Para nuestro caso, en las Instituciones de Educación Superior (IES), un proceso comprende una serie de actividades realizadas por los diferentes departamentos o servicios, que añaden valor y que ofrecen un servicio a una determinada población. En la gestión por procesos prima la visión en el cliente, sin embargo es importante aclarar que en el ámbito educativo el concepto de "cliente" tiene una dimensión diferente del que tiene un "cliente" en un ámbito comercial o empresarial, ya que en las misiones de las instituciones educativas tienen prioridad los objetivos sociales y el impacto que éstas puedan causar en su entorno. Por tanto, los "clientes" de las IES no son solamente receptores de un servicio, sino partícipes en la construcción de una sociedad que requiere sujetos capacitados, autónomos y críticos.

El objetivo de una IES en concordancia con la misión es entregar un ser social, un hombre preparado para el trabajo, para enfrentar los retos de la vida, para desarrollarse y contribuir al desarrollo de la sociedad en que vive, desde el aprender a ser, aprender a hacer, aprender a aprender y aprender a convivir, en la construcción permanente de la dignidad humana, la solidaridad colectiva y una conciencia social y ecológica, significa ante todo ser capaz de adoptar una correcta actitud frente a la vida (Manual de procesos ITM, 2007). ${ }^{7}$

Aunque esta misión podría ser ligeramente diferente en otra Institución de Educación Superior (IES), recoge elementos comunes en cuanto a preparación para la vida, para la convivencia y para la formación autónoma que caracterizan al espíritu de las IES. 
Actualmente se habla en el ámbito de las IES, de la prospectiva como una alternativa de modelo de gestión, que permite anticiparse al futuro, evitar que él nos sorprenda, y actuar en consecuencia con los intereses y necesidades institucionales y de la sociedad.

Existe un consenso en establecer que una organización exitosa moderna, con desempeño excelente como sistema global, de cualquier tipo o tamaño, tanto en el sector público como en el sector privado, que aplica la calidad total como su filosofía de administración, debe practicar la gerencia de procesos en su gestión (Mariño Navarrete, 2002).

Algunas proyecciones que se concluyen como necesarias para el perfeccionamiento de la dirección de los procesos que se llevan a cabo en las instituciones educativas, hacen referencia a la reestructuración institucional, la reformulación de planes y programas de estudio; pero sólo quien dirige, sabe que lo importante es orientarse hacia la gerencia de los procesos organizacionales como uno de los pilares de un sistema integral, holístico de planeación y gerencia, esencial para desarrollar la gerencia corporativa: "Los procesos son la arquitectura en la que está soportada una organización para entregar valor a sus clientes." (Mariño Navarrete, 2002).

La norma ISO 9000-2000 define proceso como el conjunto de actividades mutuamente relacionadas o que interactúan, las cuales transforman elementos de entrada en resultados. Los resultados en una IES pueden ser tangibles (productos) o intangibles (servicios), productos y servicios son el resultado de un proceso, por lo tanto, los procesos tienen responsables.

El proceso es un ordenamiento específico de actividades en lugar y tiempo, que tienen un principio y un fin, con insumos o entradas y productos o resultados claramente especificados para un determinado cliente o mercado. Cualquier proceso toma importancia en la medida que agregue valor a sus clientes. Si un proceso no tiene usuarios, consumidores que utilicen las salidas entregadas por éste, es un procesos cuya existencia no tiene sentido y debe desaparecer. "Todo proceso incorpora un conjunto de actividades que buscan satisfacer las necesidades del cliente" (Mejía García, 2003). 
La gerencia involucra el conocimiento de la institución en su conjunto como sistema y en capacidad de liderazgo para generar acciones de comunicación, promoción, negociación y persuasión que le permitan logro en sus objetivos, e implica el manejo estratégico de los TOG (Tecnología de la Organización y la Gerencia).

Las Instituciones de Educación Superior son empresas muy complejas, donde se realizan varios tipos de procesos. Algunos administrativos, financieros, relacionados con la educación que giran todos alrededor de los usuarios internos y externos. La gestión por procesos es una forma de organización diferente a la clásica organización funcional, y en la que prima la visión del cliente sobre las actividades de la organización. Según varios autores, los procesos así definidos son gestionados de modo estructurado y sobre su mejora se basa la de la propia organización (SESCAM-Toledo).

Este tipo de gestión ha despertado un interés creciente, siendo ampliamente utilizada por muchas organizaciones que utilizan referenciales de Gestión de Calidad y Calidad Total. El enfoque basado en procesos consiste en la identificación y gestión sistemática de los procesos desarrollados en la organización y en particular las interacciones entre tales procesos (ISO 9000:2000). La Gestión por Procesos tiene su fundamento en la construcción de modelos de los sistemas como un conjunto de procesos interrelacionados mediante vínculos causa-efecto (Rey, 2007).

La metodología utilizada consiste en la "Gerencia de Procesos", herramienta que permite examinar la dinámica de la institución, teniendo en cuenta que ésta se crea con el objeto de llevar a cabo un propósito, mediante la ejecución de una secuencia articulada de actividades. De esta manera, la Gerencia de Procesos permite alcanzar una visión sistémica de la institución, como el trascurrir continuo de una secuencia articulada de macroprocesos, procesos y procedimientos tendientes, en últimas, al logro del propósito institucional y buscando, ante todo, la coherencia entre lo que se anuncia, lo que se hace y lo que se obtiene.

Los procesos se pueden clasificar en procesos misionales, los procesos estratégicos o de direccionamiento, los procesos de apoyo 
y los procesos de evaluación. Esta clasificación, apoyada en las normas ISO 9001:2000, define como procesos misionales todos aquellos procesos que dan como resultado el cumplimiento del objeto social o razón de ser de la institución, añaden valor al cliente o inciden directamente en su satisfacción o insatisfacción, es decir, componen la cadena del valor de la organización. Los procesos estratégicos, por otro lado, son los que permiten definir y desplegar las estrategias y objetivos de la organización Son aquellos que buscan establecer visión o foco, y criterios para el alcance de logros y propósitos.

Los procesos de apoyo que son necesarios para el control y la mejora del sistema de gestión y que no puedan considerarse estratégicos ni misionales (Rey, 2007), incluyen aquellos procesos que proveen los recursos necesarios para el desarrollo de los procesos estratégicos, misionales y de evaluación; proveen servicios de utilidad para toda la Universidad en sus diferentes frentes. Y finalmente los procesos de evaluación incluyen aquellos procesos necesarios para medir y recopilar datos para el análisis del desempeño y la mejora de la eficacia y la eficiencia, y son una parte integral de los procesos estratégicos, de apoyo y los misionales, como se relacionan en la figura siguiente en el clásico texto de Michael Porter (2006) ${ }^{8}$.

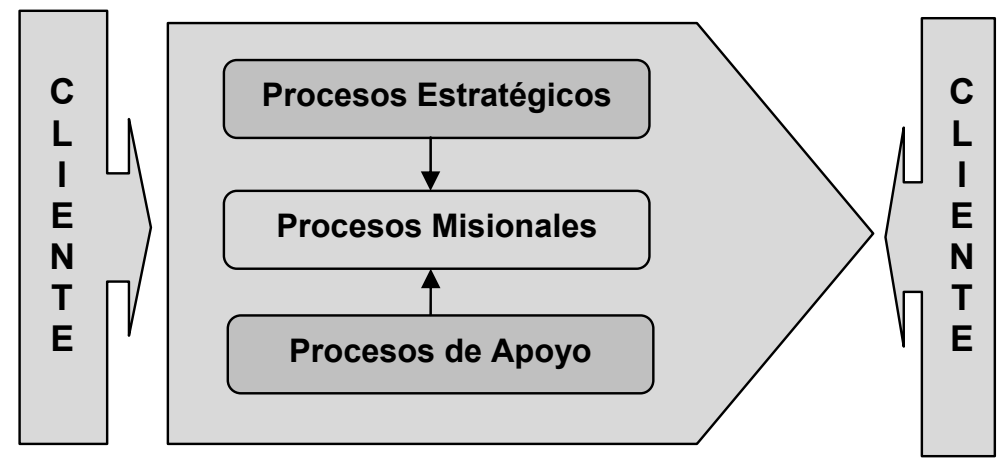

Figura 4. Cadena de valor adaptada a las ies tomada de la estructura general PROPUESTA POR Michael PoRTer (2006, ORIGINAL EN 1980)

$8 \quad$ El texto original en inglés “Competitive Strategy" se publicó en 1980. 
La cadena de valor que aparece en la figura ofrece un medio sistemático de presentar y clasificar las actividades que realiza cada sector. Las actividades de una cadena de valor de una empresa no son independientes, sino que están conectadas mediante lo que se denomina conexiones. La cadena de valor de una IES está constituida por una serie de actividades que proporcionan cadena de valor; los canales tienen cadena de valor que van pasando a través s de los cuales va pasando el producto o servicio (Porter, 2006).

Para que una IES funcione de manera eficaz, tiene que identificar y gestionar numerosas actividades relacionadas entre sí y la normatividad que las regula. Una actividad que utiliza recursos, y que gestiona con el fin de permitir que los elementos de entrada se transformen en resultados, se pueden considerar como un proceso y debe considerar el MODELO ESTÁNDAR DE CONTROL INTERNO (MECI). ${ }^{9}$

Sistema de Gestión de Calidad (SGC) se compone de un conjunto de procesos, procedimientos y actividades debidamente documentados y aplicados, con el objetivo de lograr que los servicios que ofrece una organización sean de calidad de los servicios, la satisfacción de los usuarios, el logro de buenos resultados y la reducción de costos.

Una gestión por procesos para una IES conlleva a la formación de una estructura coherente que represente el funcionamiento de la organización, que pueda hacérsele seguimiento a través de la implementación de un sistema de indicadores que permita evaluar

$9 \quad$ El Modelo Estándar de Control Interno para entidades del Estado, se genera tomando como base el artículo $1^{\circ}$ de la Ley 87 de 1993, el cual se encuentra compuesto por una serie de Subsistemas, Componentes y Elementos de Control. El Modelo Estándar de Control Interno que se establece para las entidades del Estado proporciona una estructura para el control a la estrategia, la gestión y la evaluación en las entidades del Estado, cuyo propósito es orientarlas hacia el cumplimiento de sus objetivos institucionales y la contribución de éstos a los fines esenciales del Estado. Este Modelo se ha formulado con el propósito de que las entidades del Estado obligadas puedan mejorar su desempeño institucional mediante el fortalecimiento del control y de los procesos de evaluación que deben llevar a cabo las Oficinas de Control Interno, Unidades de Auditoría Interna o quien haga sus veces. 
la eficacia y eficiencia de los procesos, tanto desde el punto de vista interno (indicadores de rendimiento) como externo (indicadores de percepción). También es necesaria la designación de responsables de proceso, que deben supervisar y mejorar el cumplimiento de todos los requisitos y objetivos del proceso asignado.

La Entidad Pública a fin de garantizar el control de cumplimiento, deberá elaborar un Normograma ${ }^{10}$ (también conocido como nomograma) con las normas de carácter constitucional, legal, reglamentario y de autorregulación que le son aplicables, verificando a través del desarrollo del Modelo Estándar de Control Interno, el cumplimiento y todas y cada una de dichas normas. En especial lo establecido con las normas NTC GP 1000:2004. Este tipo de gestión crea un esquema de relaciones para un sistema normativo sobre el cual se puede desarrollar una taxonomía normativa por procesos que abarque la organización en todas sus dimensiones, desde los procesos misionales de la misma y sobre la cual gira la organización, su gestión y control.

\section{Resultados: un ESquema de RELACIONES PARA UN SISTEMA DE INFORMACIÓN NORMATIVO}

Con los elementos jurídicos (naturaleza y jerarquía) y los conceptos de la gestión por procesos, dentro del marco de la gestión del conocimiento, se propone una construcción esquemática relacional de un sistema de información normativo para una Institución de Educación Superior.

En primera instancia, la estructura de las normas determina una jerarquía jurídica que influencia la toma de decisiones en una organización (por ejemplo una Institución de Educación Superior) ya sea de carácter público o privado. Se observa que las normas

10 El Normograma, conjunto de normas asociadas con los objetivos del proceso, sean éstas de carácter externo (Constitución Política, leyes, decretos, ordenanzas, acuerdos, resoluciones, etc.) o de carácter interno (políticas, normas administrativas, etc.) que soportan legalmente y en relación con la función de la entidad pública, la ejecución de los procedimientos. 
nacionales constitucionales son las que tienen mayor jerarquía debido a que es la constitución misma la que rige las decisiones misionales de una Nación. Consecuentemente, las normas regulatorias y reglamentarias para hacer cumplir la misión de las normas constitucionales tienen una jerarquía político administrativa en escala nacional, regional y local. Este tipo de división administrativa es común en la mayoría de los Estados contemporáneos con constitución política liberal. En la figura 5 se muestra la jerarquía de las normas en el Estado colombiano divididas en normas externas que dan las condiciones de contorno jurídico a una institución, y las normas internas que se hacen (dentro del marco jurídico externo) para lograr la misión de su naturaleza.

NORMATIVIDAD EXTERNA COLOMBIANA

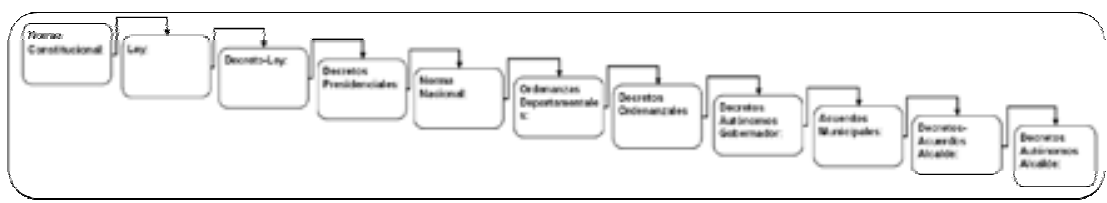

NORMATIVIDAD INTERNA DE UNA IES

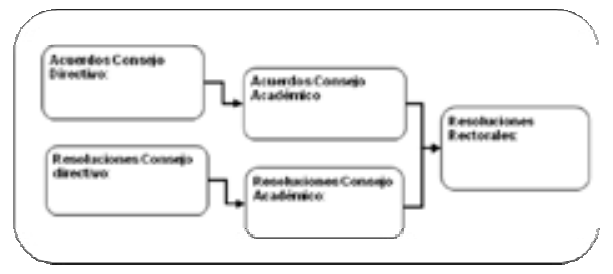

Figura 5. JERARQUÍA NORMATIVA EXTERNA E INTERNA PARA UNA IES (ELABORACIÓN PROPIA BASADA EN LA CONSTITUCIÓN POLÍTICA DE COLOMBIA DE 1991 Y NORMAS DE CONSTITUCIÓN DEL INSTITUTO TECNOLÓGICO METROPOLITANO DE MEDELLÍN)

Sin embargo, esta normatividad tal como está es difícil de hacerle seguimiento en una organización, debido a la abundancia, cantidad de emisores y cambios frecuentes. Por esto, se propone encadenar esta jerarquía normativa a una estructura organizacional por procesos que permita abarcar toda la organización y así 
mismo tener un mayor control, lograr mayores sindicadores para mejorar la gestión y la toma de decisiones.

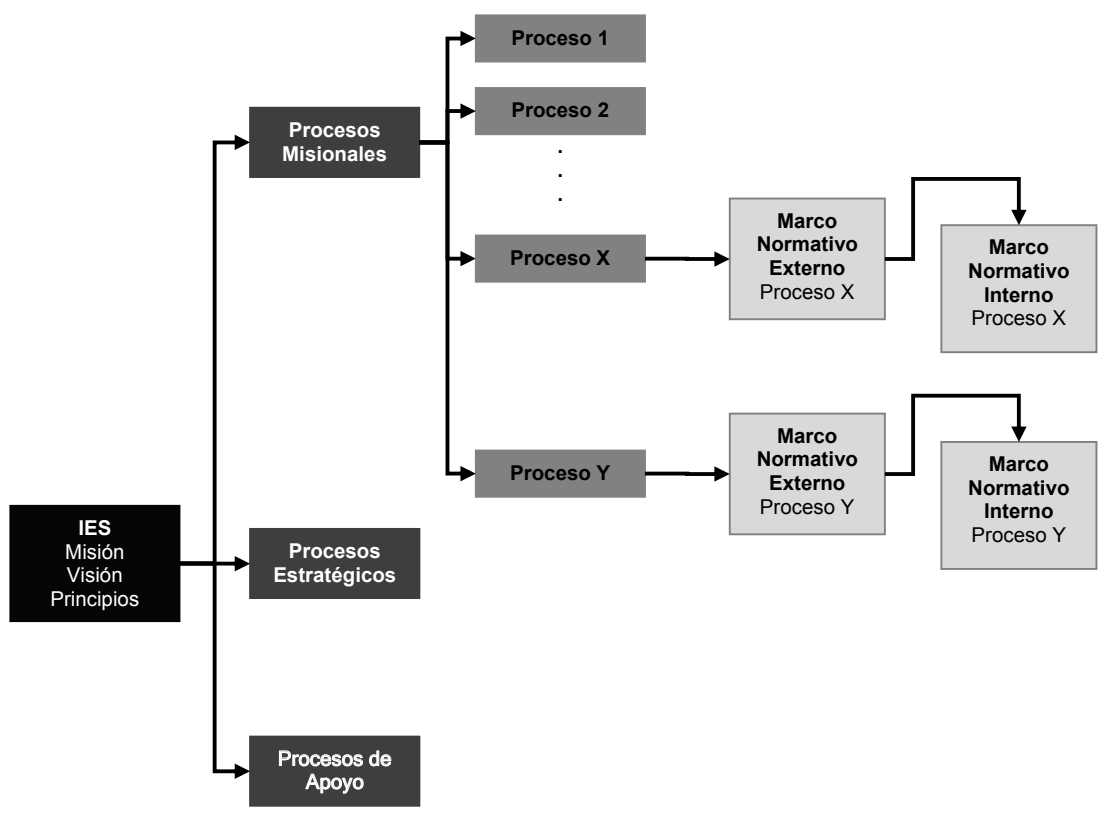

Figura 6. Relación entre los procesos de Una IES Y SU MARCo noRmativo.

(ELABORACIÓN PROPIA CON BASE EN LA GESTIÓN POR PROCESOS DE LA FAMILIA DE NORMAS ISO 9000)

Se observa en la figura 6 que en una organización cualquiera se identifican los procesos misionales, los procesos estratégicos y los procesos de apoyo (ver gestión por procesos). De allí se desprenden cada uno de los procesos necesarios para cumplir la misión de la organización de su actividad en particular. Como a cada proceso se le puede poner un marco jurídico externo e interno, de esta forma se logran barrer todas las normas de forma integral en una organización. 
Sin embargo, es necesario incluir elementos taxonómicos ${ }^{11}$ adicionales para lograr también una relación transversal de los procesos. Por esto se incluyen a continuación descriptores taxonómicos para la gestión administrativa que son relevantes en el caso de las Instituciones de Educación Superior, como el caso de "gestión académica", "gestión organizacional", "gestión financiera", "Gestión Humana” y "Control”.

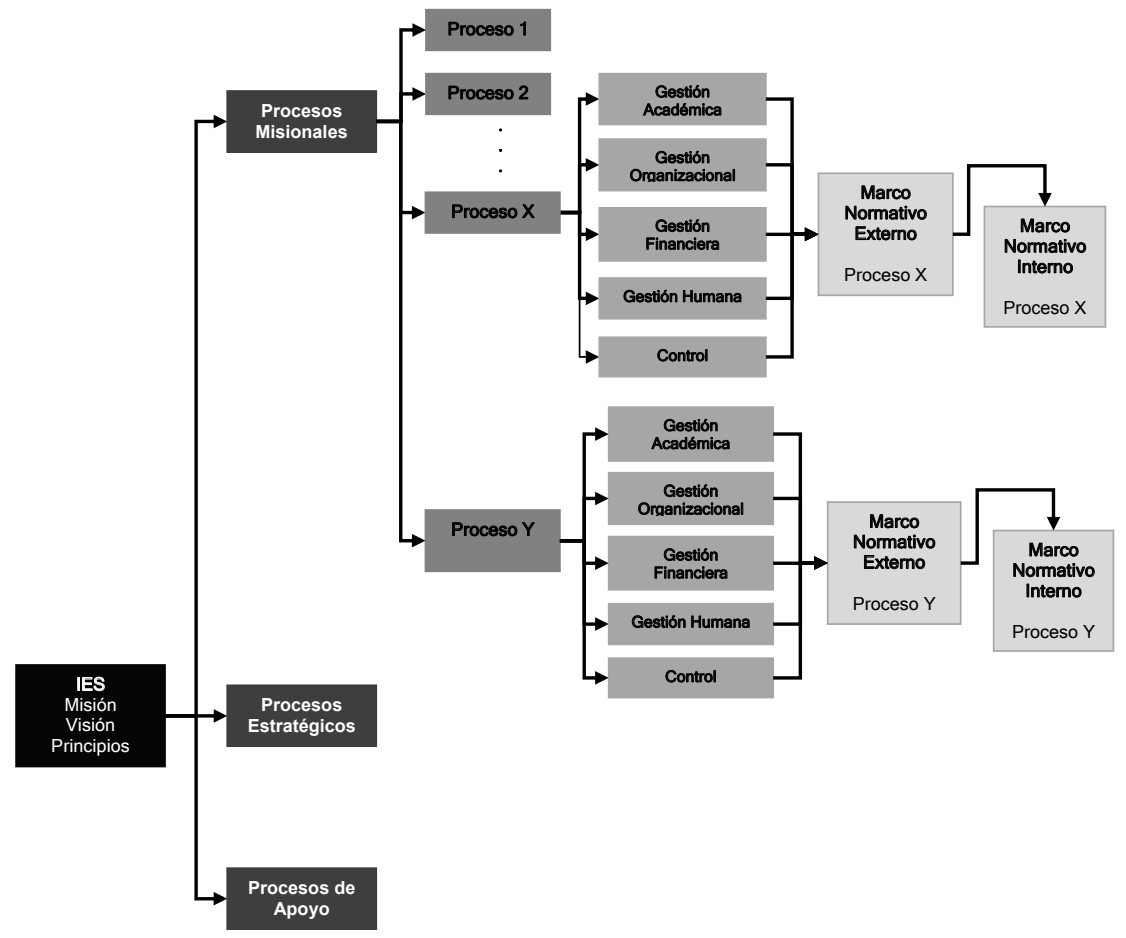

FigURA 7. ESQUEMA DE RELACIONES NORMATIVO CON TAXONES ORGANIZADO POR PROCESOS (ELABORACIÓN PROPIA CON BASE EN LA GESTIÓN POR PROCESOS DE LA FAMILIA DE NORMAS ISO 9000)

11 Llamaremos elementos taxonómicos a identificadores adicionales que recojan grupos de procesos que sean convenientes a la hora de hacer una gestión administrativa de los mismos. 
Por tanto, nuestro esquema de sistema de información normativo puede ser como se muestra en la figura 7. Aquí se observa la inclusión de "taxones" en varios niveles de gestión (académica, organizacional, financiera, humana y control) que permite una relación transversal entre los procesos.

Este esquema logra un recorrido longitudinal de la organización, así como una relación transversal entre los procesos con la inclusión de taxones por el tipo de gestión posible en dicha organización (académica, organizacional, financiera, humana y control). Cada proceso puede llevar uno o varios taxones ya que puede tener una normatividad distinta en alguna de estas dimensiones.

Algunas ventajas que surgen con esta propuesta son las siguientes:

- Se desarrolla una metodología blanda para la gestión de la información normativa en una Institución de Educación Superior. Afirmamos que una blanda en el sentido en que puede adaptarse fácilmente cada que se incluya o suprima un proceso en una organización.

- Se crea sinergia de la información emanada de las decisiones tomadas en los actos administrativos de los diferentes niveles institucionales generada por la actividad propia de la institución para su regulación y función.

- Permite un mejor control de la producción normativa, tanto interna como externa, que impacta jurídicamente en los procesos de las IES.

- Da buenas condiciones para el ordenamiento y clasificación por procesos de la normatividad producida interna y externamente en las IES.

- Facilita la efectiva toma de decisiones de la gestión interna de la organización, a partir de los cambios y nuevas regulaciones que se den en el medio jurídico en el que esté inmersa la organización.

- Ayuda a la toma de decisiones para el diseño y la implementación de la estructura organizacional y su integración. 
- Permite el diseño y desarrollo de un software para el manejo y clasificación de la información normativa de una IES según sus procesos. Como consecuencia, el diseño de los manuales de uso y aplicaciones del software para el manejo de la información normativa en una IES.

Esta propuesta de esquema de relaciones logra dar cuenta del marco normativo de cada uno de los procesos y los entes que la conforman tanto longitudinalmente (los procesos y las áreas en sí) como transversalmente (inter-procesos e inter-áreas) dentro una institución de educación superior.

\section{Conclusiones}

En primer lugar aseguramos que una organización, como un conjunto de entidades soportadas en sujetos, aprende, y ese aprendizaje surge de las relaciones internas (entre las personas que la conforman) y su entorno. Sin embargo el conocimiento de esta organización (conocimiento organizacional) no puede ser almacenado como tal, pero sí se pueden captar sus huellas (en información) que alimentan sus experiencias, modelos mentales y procedimientos.

Las normas determinan un tipo especial de entorno para una organización (el entorno jurídico); por tanto, un sistema de información normativo es una herramienta efectiva de consulta para propiciar conocimiento organizacional que surge al momento de solucionar un problema interno o con el medio. No se puede concebir un sistema de información de normas sin considerar el concepto de ordenamiento jurídico que da origen a todas las leyes y determina la jerarquía que regula los entes públicos y privados. Este ordenamiento determina el marco normativo interno y externo de cualquier institución educativa que le permite cumplir su misión dentro de los marcos legales. La ausencia del conocimiento de estas normas genera falencias en el cumplimiento misional de las instituciones para el apoyo a la gestión y al control. 
Para relacionar estas normas se dispone de los conceptos y aplicaciones de la "gestión por procesos" que permite analizar la dinámica de una institución, teniendo en cuenta que ésta se crea con un propósito: alcanzar una visión sistémica buscando ante todo la coherencia entre lo que se anuncia, lo que se hace y lo que se obtiene.

Finalmente, se puede afirmar que una Institución de Educación Superior que cuente con un modelo para la gestión de la información normativa, es una institución que está en las condiciones de generar conocimiento. Esta gestión de la información provee a los equipos de trabajo los recursos necesarios para la solución de problemas en el menor tiempo posible. En este caso particular, el esquema propuesto logra una infraestructura para la gestión del conocimiento que permite la búsqueda de soluciones rápidas a los problemas que enfrentan las instituciones. Esto permite crear condiciones para una cultura organizacional a través del diseño de estrategias y de la aplicación de políticas dirigida al uso racional, efectivo y eficiente de la información normativa en función de su misión y de los objetivos.

\section{Bibliografía}

Alavi, M.; Leider, D. (1999), Knowledge Management and knowledge Management Systems: Conceptual Foundations and Research Issues, working papers, INSEAD R\&D 99/34/TM.

Argentina, Ministerio de educación. INET. Colección /las tecnologías de organización y gestión se instalan en la escuela. Art. No. 5. Buenos Aires república Argentina.

Bustelo C., Amarilla R., (2001) Gestión del conocimiento y gestión de información. Boletín del Instituto Andaluz de Patrimonio Histórico 2001; VIII (34): 226-230.

Cañedo Andalia R. (1997) Elementos conceptuales útiles para la implementación de los sistemas de calidad. ACIMED: 5 Supl: 15.

Colombia MECI 1000:2005 según ley 87/1993. Congreso de la República de Colombia. 
Colombia NTC GP1000:2004 Norma de calidad para entidades públicas. Instituto Colombiano de Normas Técnicas ICONTEC.

Colombia, Ley 872 de 2003. Por la cual se crea el sistema de gestión de la calidad en la Rama Ejecutiva del Poder Público y en otras entidades prestadoras de servicios. Congreso de la República.

Correa, Guillermo (2007). Gerencia, Administración, Gestión del Conocimiento en las Organizaciones. Conferencia para el Instituto Tecnológico Metropolitano de Medellín. Noviembre de 2007.

Hanson, N. R. (1977) Patrones de Descubrimiento en: Observación. Madrid Alianza 1977, pp. 77-112.

Instituto Tecnológico Metropolitano (ITM, 2007). Manual de procesos. Oficina de Planeación. Editorial ITM, Medellín.

ISO 9000-2000. Directrices para la selección y utilización de las normas para la gestión de la calidad y el aseguramiento de la calidad. Ginebra: ISO; 2000 .

ISO 9001-2000. Sistemas de gestión de la calidad. Requisitos. Ginebra: ISO; 2000.

ISO 9004-2000. Sistema de gestión de la calidad. Directrices para la mejora del desempeño. Ginebra: ISO; 2000.

Koontz, H. y Weihrich H. Administración una Perspectiva global. Duodécima edición. Ed. McGraw-Hill.

Mariño Navarrete, Hernando, (2002) Gerencia de procesos, Alfa Omega Grupo Editor S.A. de C.V.

Martínez Roldán, Luis (2005). Curso de teoría del Derecho. España: Editorial Ariel, 2005.

Mejía García, Braulio MD-MSP, (2003) Gerencia de procesos para la organización y el control interno de empresas de salud. ECOE Ediciones.

Nonaka I, Takeuchi H. (1995) The Knowledge Creating Company: How Japanese Companies Create the Dynamics of Innovation? Oxford University Press, New York.

Polanyi, Michael (1966). El Estudio del Hombre. Edición española de Antonio Cucurullo, Paidós S.A.I.C.F., Buenos Aires.

Ponjuán Dante (2004): Gestión de la información: Dimensiones e implementación para el éxito organizacional. $1^{\circ}$ Ed.: Nuevo Paradigma, Rosario 2004. 
Ponjuán Dante, Gloria (1998). Gestión de la Información en las Organizaciones: Principios, Conceptos o Aplicaciones. Talleres de Impresos Universitaria. Santiago de Chile, 1998.

Porter, Michael. (2006) Estrategia y ventaja competitiva, Ediciones Deusto.

Rey, Domingo. "La Gestión Tradicional y la Gestión por Procesos". Gestiópolis., disponible en [http://www.gestiopolis.com/recursos4/docs/ger/gestitra.htm]. Consultado el 10 de mayo de 2007.

Ruiz Callejas, José Manuel (2004). Dirección y Gestión Educativa. Colección autores Sumer, serie formación.

Santos A., Piedrafita J., Marañón B. Ontología para la Gestión del Conocimiento. Documento en línea disponible en [http://es.geocities.com/ontologia04/definicion.htm]. Consultado el 10 de mayo de 2007.

Soto Balbón Ma, Barrios Fernández NM (2006). Gestión del conocimiento. Parte I. Revisión crítica del estado del arte. Acimed 2006; 14(2).

Toledo. Sescam (2002): Servicio de Calidad de la Atención Sanitaria. Gestión por Procesos. 2002. 21 p. 